\title{
Association of Phase Angle and Sarcopenia in Chronic Msculoskeletal Pain Patients: A Retrospective Study
}

Hironori Tsuji

Okayama Red Cross General Hospital

Tomoko Tetsunaga ( $\square$ kwdtmk1201@yahoo.co.jp )

Okayama University Hospital

Tomonori Tetsunaga

Okayama University Hospital

Haruo Misawa

Okayama University Hospital

Masanori Hamada

Okayama University Hospital

Yoshimi Katayama

Okayama University Hospital

Kentaro Yamane

Okayama Medical Center

Yoshiaki Oda

Okayama University Hospital

Shinichiro Takao

Okayama University

Yuya Kajiki

Okayama University

Yoshitaka Takei

Kurashiki Municipal Hospital

Keiichiro Nishida

Okayama University Hospital

Masuo Senda

Okayama University Hospital

Toshifumi Ozaki

Okayama University 
Keywords: sarcopenia, chronic musculoskeletal pain, phase angle, detection, bioimpedance analysis Posted Date: May 6th, 2021

DOI: https://doi.org/10.21203/rs.3.rs-461811/v1

License: (c) (1) This work is licensed under a Creative Commons Attribution 4.0 International License. Read Full License 
1 Association of phase angle and sarcopenia in chronic musculoskeletal pain patients: a

2

retrospective study

4 Hironori Tsuji, M.D., Ph.D. ${ }^{a}$, Tomoko Tetsunaga, M.D., Ph.D. ${ }^{\text {b }}{ }^{*}$, Tomonori Tetsunaga,

5 M.D., Ph.D. ${ }^{\text {c }}$, Haruo Misawa, M.D., Ph.D. ${ }^{\mathrm{b}}$, Masanori Hamada, M.D., Ph.D. ${ }^{\mathrm{d}}$, Yoshimi

6 Katayama, M.D., Ph.D. ${ }^{\mathrm{d}}$, Kentaro Yamane, M.D., Ph.D. ${ }^{\mathrm{e}}$, Yoshiaki Oda, M.D., Ph.D. ${ }^{\mathrm{b}}$,

7 Shinichiro Takao, M.D. ${ }^{\mathrm{f}}$, Yuya Kajiki, M.D. ${ }^{\mathrm{f}}$, Yoshitaka Takei, M.D., Ph.D. ${ }^{\mathrm{g}}$, Keiichiro

8 Nishida, M.D., Ph.D. ${ }^{\text {c }}$, Masuo Senda, M.D., Ph.D. ${ }^{d}$, Toshifumi Ozaki, M.D., Ph.D. ${ }^{f}$

10 a Department of Orthopedic Surgery, Okayama Red Cross Hospital, Okayama, Japan, 2-1-1

11 Aoe, Kitaku, Okayama City, Okayama 700-8607, Japan

12

${ }^{\mathrm{b}}$ Department of Orthopedic Surgery, Okayama University Hospital, Okayama, Japan, 2-5-1,

13 Shikata-cho, Kitaku, Okayama City, Okayama 700-8558, Japan

$14{ }^{\mathrm{c}}$ Department of Locomotive Pain Center, Okayama University Hospital, Okayama, Japan, 2-

15 5-1, Shikata-cho, Kitaku, Okayama City, Okayama 700-8558, Japan

${ }^{\mathrm{d}}$ Department of Rehabilitation Medicine, Okayama University Hospital, Okayama, Japan, 2-

17 5-1, Shikata-cho, Kitaku, Okayama City, Okayama 700-8558, Japan

${ }^{e}$ Department of Orthopedic Surgery, Okayama Medical Center, Okayama, Japan, 1711-1, 
$20{ }^{\mathrm{f}}$ Department of Orthopedic Surgery, Okayama University Graduate School of Medicine,

21 Dentistry, and Pharmaceutical Sciences, Okayama, Japan, 2-5-1 Shikata-cho, Kitaku,

22 Okayama City, Okayama 700-8558, Japan

$23{ }^{\mathrm{g}}$ Department of Orthopedic Surgery, Kurashiki Municipal Hospital, Okayama, Japan, 2-39,

24 Kojima-ekimae, Kurashiki City, Okayama 711-0921, Japan

25

$26 *$ Corresponding Author: Tomoko Tetsunaga, M.D., Ph.D.

27 Department of Orthopedic Surgery, Okayama University Hospital, Okayama, Japan, 2-5-1

28 Shikata-cho, Kitaku, Okayama City, Okayama 700-8558, Japan

29 Tel: 81-86-235-7273

30 Fax: 81-86-223-9727

$31 \quad$ E-mail: kwdtmk1201@yahoo.co.jp 


\section{Abstract}

33 In chronic musculoskeletal pain patients, early prevention and detection for musculoskeletal

34 dysfunction (i.e., sarcopenia) is an issue of significant clinical interest. Phase angle, which can be measured through bioelectrical impedance analysis, can detect sarcopenia; however, the evidence on chronic musculoskeletal pain patients is limited. This study aimed to assess the relationship between phase angle and sarcopenia in patients with chronic musculoskeletal pain.

A total of 190 patients (51 men, 139 women; mean age: 67.2 years) with chronic musculoskeletal pain were included in this retrospective cross-sectional study. Sarcopenia was diagnosed using the Asian Working Group for Sarcopenia Criteria 2019. A total of 51 patients (26.7\%) including 10 men (19.6\%) and 41 women (29.5\%) were diagnosed with sarcopenia. Phase angle, sarcopenia-related factors, age, and BMI differed significantly in patients with and without sarcopenia. On multiple logistic regression analysis, the prevalence of sarcopenia was

44 observed to be significantly correlated with phase angle and BMI. The areas under the curve exhibited high accuracy in discriminating sarcopenia in men and moderate accuracy in both genders and in women. We conclude that phase angle may be a useful tool for detecting sarcopenia in the patients with chronic musculoskeletal pain.

Keywords: sarcopenia, chronic musculoskeletal pain, phase angle, detection, bioimpedance 


\section{Introduction}

51 Chronic pain, which affects $20 \%$ of the general population, is a global problem that decreases

52 activities of daily living. ${ }^{1,2}$ Meanwhile, $30-50 \%$ of older adults suffer with chronic pain, mostly

53 originating in the musculoskeletal system. ${ }^{3}$ Furthermore, musculoskeletal conditions are the

54 leading cause of disability and also have a large impact on many other aspects of older people's

55 health, such as low physical activity level, poor mobility, frailty, depression, cognitive

56 impairment, and falls. ${ }^{4}$ In chronic musculoskeletal pain patients, physical function and activity

57 are impaired from an early age, and as decreased activity exacerbates and intracts the pain itself,

58 early prevention and detection for musculoskeletal dysfunction is an issue of significant clinical

59 interest. ${ }^{5}$ Sarcopenia, a progressive and generalized musculoskeletal disorder, has attracted

60 attention $^{6-8}$ and is associated with increased adverse outcomes, including falls, fractures,

61 functional decline, and mortality. ${ }^{9-13}$ While chronic musculoskeletal pain and sarcopenia may

62 be correlated and the early detection of sarcopenia may be important in patients with chronic

63 pain, evidence on this correlation is limited.

64 Conversely, phase angle, which can be measured noninvasively through bioelectrical

65 impedance analysis, is reported to reflect the quality of cells, and a lower phase angle suggests

66 decreased cellular integrity. ${ }^{14-16}$ Previous reports have correlated phase angle with nutritional

67 status, muscle strength, and mortality. ${ }^{17-19}$ Moreover, phase angle can be used to detect

68 sarcopenia. ${ }^{20-24}$ However, it is unclear whether phase angle is lower and can detect sarcopenia 
69 in chronic musculoskeletal pain patients. Therefore, this study aimed to assess the relationship

70 between phase angle and sarcopenia in patients with chronic musculoskeletal pain.

71

\section{Results}

73 Participants' characteristics

74 The characteristics of the patients are shown in Table 1. The mean age was 67.2 years (standard

75 deviation [SD]: 13.5) and mean Numeric Rating Scale (NRS) score was 5.2 points (SD: 2.5).

76 Sarcopenia was diagnosed in 51 (26.7\%), 10 (19.6\%), and 40 (29.5\%) patients of all, male, and

77 female patients, respectively. The mean phase angle was 4.7 degrees (SD: 1.0). Pain site and

78 other sarcopenia-related factors are also shown on Table 1.

79

80 Table 1. Participants characteristics

\begin{tabular}{lccc}
\hline \multicolumn{1}{c}{ Variables } & All $(\mathrm{n}=190)$ & Men $(\mathrm{n}=51)$ & Women $(\mathrm{n}=139)$ \\
\hline Age (years) & $67.2 \pm 13.5$ & $65.3 \pm 12.2$ & $67.9 \pm 13.9$ \\
BMI $\left(\mathrm{kg} / \mathrm{m}^{2}\right)$ & $23.6 \pm 4.5$ & $23.7 \pm 3.7$ & $23.5 \pm 4.8$ \\
Sarcopenia & $51(26.7)$ & $10(19.6)$ & $41(29.5)$ \\
Grip power $(\mathrm{kg})$ & $19.6 \pm 10.4$ & $29.3 \pm 12.0$ & $16.1 \pm 6.9$ \\
Gait speed $(\mathrm{m} / \mathrm{s})$ & $1.1 \pm 0.4$ & $1.1 \pm 0.4$ & $1.3 \pm 0.4$ \\
SMI $\left(\mathrm{kg} / \mathrm{m}^{2}\right)$ & $6.7 \pm 1.3$ & $7.9 \pm 1.1$ & $6.1 \pm 0.9$
\end{tabular}




$\begin{array}{lccc}\text { Phase angle }\left(^{\circ}\right) & 4.6 \pm 0.9 & 5.3 \pm 0.9 & 4.4 \pm 0.8 \\ \text { NRS (points) } & 5.2 \pm 2.5 & 4.9 \pm 2.8 & 5.3 \pm 2.4 \\ \text { Pain site } & & & \\ \text { Cranio-cervical } & 32(16.8) & 11(21.6) & 21(15.1) \\ \text { Upper limb } & 41(21.5) & 15(29.4) & 26(18.7) \\ \text { Trunk } & 111(58.1) & 27(52.9) & 84(60.4) \\ \text { Lower limb } & 98(51.3) & 25(49.0) & 73(52.5)\end{array}$

81 Data are expressed as mean \pm standard deviation for continuous variables, and as percentages

82 (\%) for categorical variables. BMI, body mass index; SMI, skeletal mass index; NRS,

83 numeric rating scale.

84

85

86 Correlations among phase angle and measured variable

87 Table 2 shows the correlation among phase angle and other variables. Phase angle was

88 significantly correlated with age $(\mathrm{r}=-0.54, p<0.001)$, BMI $(\mathrm{r}=0.21, p=0.003)$, grip power

$89(\mathrm{r}=0.61, p<0.001)$, gait speed $(\mathrm{r}=0.42, p<0.001)$, skeletal muscle index (SMI) $(\mathrm{r}=0.71, p$

$90<0.001)$, and NRS $(\mathrm{r}=-0.19, p=0.007)$.

91

92 Table 2. Correlation among phase angle and measured parameters 


\begin{tabular}{ccc}
\hline & \multicolumn{2}{c}{ Phase angle } \\
Variables & $\mathrm{r}$ & $p$ \\
Age & -0.54 & $<0.001$ \\
BMI & 0.21 & 0.003 \\
Grip power & 0.61 & $<0.001$ \\
Gait speed & 0.42 & $<0.001$ \\
SMI & 0.71 & $<0.001$ \\
NRS & -0.19 & 0.01 \\
\hline
\end{tabular}

93 BMI, body mass index; SMI, skeletal mass index; NRS, numeric rating scale

\section{Comparison between patients with and without sarcopenia}

97 Table 3 shows the characteristics of patients with and without sarcopenia. All sarcopenia-

98 related factors, phase angle, age, and body mass index (BMI) differed significantly in patients

99 with and without sarcopenia. Multiple logistic regression analysis revealed a significant

100 correlation between the prevalence of sarcopenia and phase angle (odds ratio $[\mathrm{OR}]=0.09, p<$

$1010.001)$ and BMI $(\mathrm{OR}=0.80, p<0.001)($ Table 4$)$.

102

103 Table 3. Participants characteristics with and without sarcopenia 


\section{With sarcopenia Without sarcopenia}

Variables

$$
(\mathrm{n}=51) \quad(\mathrm{n}=139)
$$

Age (years)

$74.3 \pm 11.3$

$64.6 \pm 13.4$

$<0.001$

Gender (men/women)

$10 / 41$

$41 / 98$

0.199

$\begin{array}{cccc}\text { BMI }\left(\mathrm{kg} / \mathrm{m}^{2}\right) & 21.2 \pm 3.3 & 24.4 \pm 4.6 & <0.001 \\ \text { Grip power }(\mathrm{kg}) & 12.4 \pm 5.4 & 22.3 \pm 10.5 & <0.001 \\ \text { Gait speed }(\mathrm{m} / \mathrm{s}) & 0.9 \pm 0.4 & 1.2 \pm 0.4 & <0.001 \\ \text { SMI }\left(\mathrm{kg} / \mathrm{m}^{2}\right) & 5.3 \pm 0.5 & 7.1 \pm 1.1 & <0.001 \\ & 3.9 \pm 0.6 & 4.9 \pm 0.8 & <0.001 \\ \text { Phase angle }\left(^{\circ}\right) & 5.7 \pm 2.3 & 5.0 \pm 2.6 & 0.076\end{array}$

104 Data are expressed as mean \pm standard deviation for continuous variables. BMI, body mass

105 index; SMI, skeletal mass index; NRS, numeric rating scale.

106

107

108 Table 4. Multiple logistic regression analysis examining factors associated with the

109 sarcopenia

95\% CI

\begin{tabular}{ccccc}
\multirow{2}{*}{ Variables } & Odds Ratio & \multicolumn{2}{c}{$95 \%$ CI } & \multirow{2}{*}{$\boldsymbol{P}$-value } \\
\cline { 3 - 4 } & 0.09 & Lower & Upper & \\
\hline Phase angle & 0.03 & 0.23 & 0.000
\end{tabular}


$110 \mathrm{CI}$, confidence interval; BMI, body mass index.

111

112

\section{The discrimination capacity of phase angle for sarcopenia}

114 The discrimination value of phase angle for sarcopenia was assessed by the receiver operating

115 characteristic (ROC) curves (Figure 1). The areas under the curve (AUC) for both genders, for

116 men, and for women were $0.851,0.911$, and 0.837 , respectively. The cut-off value as calculated

117 by Youden index for both genders, for men, and for women were 4.2, 5.1, and 4.2, respectively

118 (Table 5). 
A
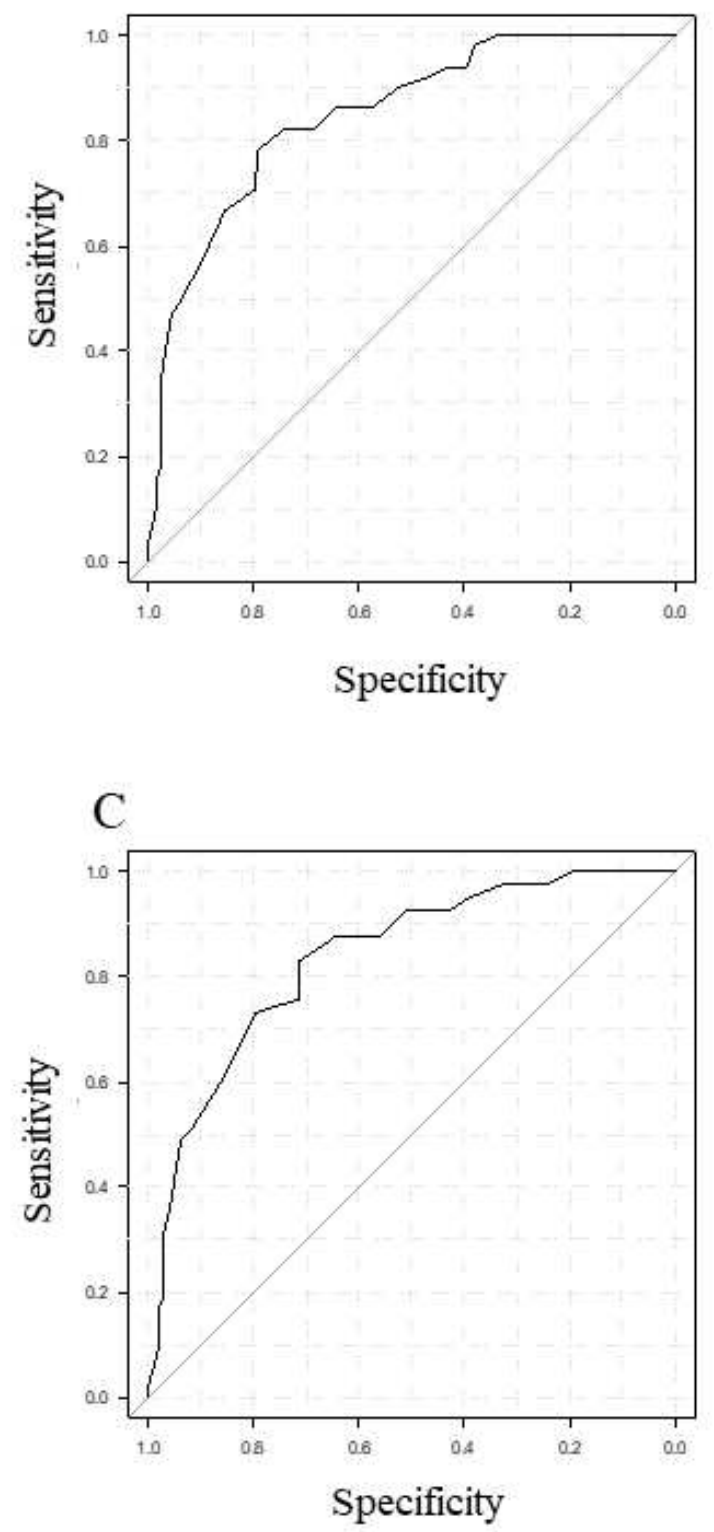

\section{B}

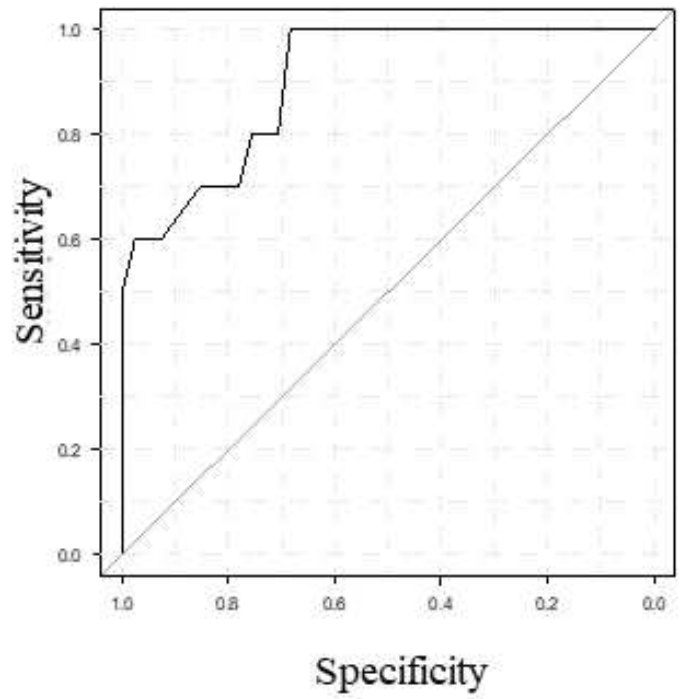

Specificity

119 Fig. 1. The receiver operating characteristic curves of phase angle for discriminating

120 sarcopenia in terms of gender: (A) both genders, (B) men, and (C) women.

122 Table 5. AUCs and cut-off values of phase angle discriminating sarcopenia.

AUC Cut-off value Sensitivity, \% Specificity, \% 


\begin{tabular}{ccccc}
\hline All & $0.85(0.79-0.91)$ & 4.2 & 78.4 & 79.1 \\
Men & $0.90(0.81-1.00)$ & 5.1 & 100 & 68.3 \\
Women & $0.84(0.77-0.91)$ & 4.2 & 82.9 & 71.4 \\
\hline
\end{tabular}

123 AUC, area under the curve. Values within parentheses show 95\% confidence intervals. The

124 sensitivity and specificity of the cut-off values are shown.

\section{Discussion}

128 Our findings showed that in chronic musculoskeletal pain patients, the phase angle significantly

129 correlated with age, BMI, grip power, gait speed, SMI, and NRS, and was lower in patients

130 with sarcopenia than in those without it. The lower phase angle was significantly correlated

131 with sarcopenia, showing a high accuracy in discriminating sarcopenia in men and moderate

132 accuracy in both genders and in women.

133 For the treatment of chronic pain, a theory of the Fear-avoidance model, that explains pain

134 chronicity, is important. ${ }^{25-27}$ In this model, pain causes fear, avoidance, and eventually

135 immobilization and disuse syndrome, which exacerbate the pain itself. Therefore, it is very

136 important to perform physical exercise and maintain daily activities for the treatment of chronic

137 musculoskeletal pain. ${ }^{28,29}$ In this study, about $20 \%$ of men and $30 \%$ of women among patients

138 with chronic musculoskeletal pain exhibited sarcopenia. In these patients, another vicious spiral, 
139 that reduces muscle mass may cause fatigue in daily activities, resulting in greater

140 immobilization. Therefore, patients with sarcopenia may be intractable in terms of chronic pain

141 and special attention should be paid to the treatment of sarcopenia patients with chronic

142 musculoskeletal pain.

143 Previous reports have suggested that phase angle was lower in individuals with sarcopenia, and

144 several possible causes were reported to explain it. One of the most commonly reported causes

145 was nutrition, and patients with low phase angle were considered as having malnutrition. ${ }^{20}$

146 Previous reports that had considered BMI as a nutritional indicator reported that as patients with

147 sarcopenia showed lower phase angle and BMI, and were diagnosed with malnutrition. ${ }^{24}$ In our

148 study, as multivariate analysis adjusted for age, gender, and degree of pain, low phase angle

149 and BMI were significantly correlated with the prevalence of sarcopenia. Therefore, sarcopenia

150 patients with chronic musculoskeletal pain were diagnosed with malnutrition, which would

151 suggest the need for nutrition therapy. Other reasons which should be considered is that phase

152 angle correlated with functional status or muscle quality. ${ }^{21}$ In our study, phase angle was

153 significantly correlated with physical function and muscle strength, and phase angle of the

154 patients with sarcopenia was lower than of those without it, which was similar to the results of

155 previous reports.

156 As for the treatment of chronic musculoskeletal pain, we think that prevention of sarcopenia is

157 very important, and detecting sarcopenia plays an important role in daily clinical practice. 
158 Previous reports suggested that phase angle was useful for detecting sarcopenia. ${ }^{20}$ In addition,

159 the AUC in ROC curves was 0.73 in kidney transplant recipients, ${ }^{24}$ and 0.718 for men and 0.721

160 for women in community-dwelling individuals $;{ }^{21}$ and 0.85 in our study, which was similar to

161 the results of previous studies. As for cut-off point of phase angle, previous reports suggested

162 the following: 4.46 for kidney transplant recipients, ${ }^{24} 5.05$ for cirrhosis,${ }^{23} 4.55$ for community-

163 dwelling and hospitalized older adults, ${ }^{22} 4.05$ for men and 3.55 for women in community-

164 dwelling individuals ${ }^{21}$ for discriminating sarcopenia. Similarly, our results recommended the

165 following cut-off points: 4.2 for both genders, 5.1 for men, and 4.2 for women with chronic

166 musculoskeletal pain. Therefore, our study demonstrated that phase angle was also useful for

167 detecting sarcopenia in chronic musculoskeletal pain patients.

168 As this study contributed new findings, there were several limitations. First, as this study was

169 conducted on Japanese patients and we used the Asian Working Group for Sarcopenia (AWGS)

1702019 criteria for sarcopenia diagnosis, the results may differ for those involving other

171 populations and those using other sarcopenia criteria such as those proposed by the European

172 working group for sarcopenia in older people ${ }^{30}$ or the international working group on

173 sarcopenia. ${ }^{31}$ Second, it was known that psychosocial factors were intricately intertwined in

174 patients with chronic musculoskeletal pain, and such items were not considered in this study.

175 Third, as this study was a retrospective cross-sectional study, it was difficult to evaluate

176 chronological order or change among phase angle, sarcopenia, and chronic musculoskeletal 
177 pain. With these limitations, further prospective studies which include more detailed

178 assessments and countries would be needed to explore the relationships among phase angle,

179 sarcopenia, and chronic musculoskeletal pain.

180 In conclusion, this study demonstrated that in chronic musculoskeletal pain patients, phase

181 angle was significantly correlated with prevalence of sarcopenia, and showed high accuracy in

182 discriminating sarcopenia in men and moderate accuracy in both genders and in women. Phase

183 angle may be useful in detecting sarcopenia among patients with chronic musculoskeletal pain.

185 Methods

\section{Study Participants}

187 This retrospective study was conducted at Okayama University Hospital and Kurashiki

188 Municipal Hospital. The participants included 190 patients (51 men and 139 women) with

189 chronic musculoskeletal pain who visited our pain outpatient clinic between June 2019 and

190 February 2021. The inclusion criteria for this study were the following: (i) age over 40 years,

191 (ii) pain for longer than 3 months, and (iii) complete self-report questionnaires and physical

192 examination. The exclusion criteria were as follows: (i) ongoing litigation, (ii) dementia, (iii)

193 delirium, or (iv) other conditions that made completion of questionnaires and physical

194 examinations difficult. The institutional review board of Okayama University Hospital

195 approved this study (approval number 2104-035) and the need for patient informed consent was 
196 waived due to its retrospective design. This study was conducted in accordance with the Code

197 of Ethics of the World Medical Association (Declaration of Helsinki) for experiments involving 198 humans.

\section{Diagnosis of sarcopenia}

201 Diagnosis of sarcopenia was performed according to the AWGS Criteria 2019. ${ }^{32}$ Gait speed,

202 grip strength, and muscle mass were used in this study. The criteria for low muscle strength

203 was handgrip strength $<28 \mathrm{~kg}$ for men and $<18 \mathrm{~kg}$ for women, and the criteria for low physical

204 performance was 6-m walking speed of $<1.0 \mathrm{~m} / \mathrm{s}$. The SMI was assessed using Inbody 770 and

205 S10 (InBody Japan, Tokyo, Japan), and low muscle mass was defined by an SMI $<7.0 \mathrm{~kg} / \mathrm{m}^{2}$ in

206 men and $<5.7 \mathrm{~kg} / \mathrm{m}^{2}$ in women. Sarcopenia was defined by the presence of low muscle mass

207 and either low muscle strength or low physical performance.

\section{Assessment of phase angle}

210 Phase angle was defined by the following equation using $50-\mathrm{kHz}$ current:

211 Phase angle $($ degrees $)=\operatorname{arctangent}($ reactance $(\mathrm{Xc}) /$ resistance $(\mathrm{R})) \times(180 / \pi)$

212 This calculation was performed automatically in the device, and we used the data which was

213 obtained during SMI assessment. 


\section{Pain intensity assessment}

216 The NRS was used for assessment of pain intensity. NRS scores ranged from 0 to 10 , with 0

217 representing no pain and 10 representing the worst imaginable pain. ${ }^{33}$ The average pain 218 intensity in the past one week was used in this study.

220 Statistical analyses

221 Descriptive statistics were presented as mean \pm SD for continuous variables and as numbers

222 and percentages for categorical variables. The Kolmogorov-Smirnov test was used to assess

223 normality for continuous variables. We analyzed correlations of phase angle with each

224 measured variable by using Spearman's rank correlation coefficient. Then, we performed the

225 Mann-Whitney U test to compare the measured parameters in patients with and without

226 sarcopenia. In a subsequent analysis, we performed multiple logistic regression analysis to

227 evaluate the factors and OR associated with sarcopenia, and the explanatory variables included

228 phase angle, body mass index, age, and gender. Next, to evaluate the discrimination

229 performance of phase angle, AUC was calculated by using a ROC analysis. Then, the sensitivity

230 and specificity were calculated by using the best cut-off point of phase angle for both and each

231 gender with the Youden index for the ROC, respectively. For the statistical analyses, we used

232 EZR software (Saitama Medical Center Jichi Medical University, Tochigi, Japan), which is a 
233 graphical user interface for $\mathrm{R}$ (The R Foundation for Statistical Computing). Results were

234 considered significant for $p<0.05$.

235

236 Acknowledgments

237 The authors would like to thank all the medical staff who collaborated this study.

238

239 Authors' contributions

240 Conceptualization: H. T. and Tomoko T. Methodology: H. T. and Tomoko T. Formal analysis

241 and investigation: H. T. and Tomonori T. Writing - original draft preparation: $\mathrm{H}$. T., Tomoko

242 T. and Tomonori T. Writing - review and editing: H. M., K. Y. and Y. O. Funding acquisition:

243 Tomoko T. Resources: M. S., M. H., Y. Katayama, Tomoko T., S. T., Y. Kajiki, and Y. T.

244 Supervision: K. N., M. S. and T. O.

245

246 Financial support

247 This study was supported by JSPS KAKENHI Grant Number 17K16691.

248

249 Conflicts of interest

250 The authors have no conflicts of interest to report. 
252 1. Blyth, F. M. et al. Chronic pain in Australia: a prevalence study. Pain 89(2), 127-134 (2001). 10.1016/S0304-3959(00)00355-9

2. Breivik, H., Collett, B., Ventafridda, V., Cohen, R. \& Gallacher, D. Survey of chronic pain in Europe: prevalence, impact on daily life, and treatment. Eur. J. Pain (London, England) 10(4), 287-333 (2006). 10.1016/j.ejpain.2005.06.009, Pubmed:16095934

3. Dieppe, P. Chronic musculoskeletal pain. BMJ (Clin. Res. Ed 346, f3146 (2013).

4. Blyth, F. M. \& Noguchi, N. Chronic musculoskeletal pain and its impact on older people. Best Pract. Res. Clin. Rheumatol. 31(2), 160-168 (2017). 10.1016/j.berh.2017.10.004, Pubmed:29224694

5. Tsuji, H. et al. Cognitive factors associated with locomotive syndrome in chronic pain patients: A retrospective study. J. Orthop. Sci. (2020). 10.1016/j.jos.2020.08.007, Pubmed: $\underline{32943300}$

265 6. Evans, W. J. \& Campbell, W. W. Sarcopenia and age-related changes in body composition and functional capacity. J. Nutr. 123(2) Suppl, 465-468 (1993). $\underline{10.1093 / \mathrm{jn} / 123 . \operatorname{suppl} 2.465}$, Pubmed:펴29405

268 7. Rosenberg, I. H. Sarcopenia: origins and clinical relevance. J. Nutr. 127(5) Suppl, 990S-991S (1997). 10.1093/jn/127.5.990S, Pubmed:1164280 
270 8. Dos Santos, L., Cyrino, E. S., Antunes, M., Santos, D. A. \& Sardinha, L. B.

271

272

273

274

275

276

277

278

279

280

281

282

283

284

285

286

287

288
Sarcopenia and physical independence in older adults: the independent and synergic

role of muscle mass and muscle function. J. Cachexia Sarcopenia Muscle 8(2), 245250 (2017). 10.1002/jcsm.12160, Pubmed:27897417

9. Landi, F. et al. Sarcopenia as a risk factor for falls in elderly individuals: results from the ilSIRENTE study. Clin. Nutr. (Edinburgh, Scotland) 31(5), 652-658 (2012).

$\underline{10.1016 / j . c l n u .2012 .02 .007, \text { Pubmed:22414775 }}$

10. Wong, R. M. Y. et al. The relationship between sarcopenia and fragility fracture-a systematic review. Osteoporos. Int. J. Established Result Coop. Between Eur. Found. Osteoporos. Natl Osteoporos. Found. USA 30(3), 541-553 (2019). 10.1007/s00198-

\section{8-04828-0, Pubmed: $\underline{30610245}$}

11. Zhang, Y., Hao, Q., Ge, M. \& Dong, B. Association of sarcopenia and fractures in community-dwelling older adults: a systematic review and meta-analysis of cohort studies. Osteoporos. Int. J. Established Result Coop. Between Eur. Found. Osteoporos. Natl Osteoporos. Found. USA 29(6), 1253-1262 (2018). 10.1007/s00198-

\section{8-4429-5, Pubmed:29500527}

12. Yeung, S. S. Y. et al. Sarcopenia and its association with falls and fractures in older adults: A systematic review and meta-analysis. J. Cachexia Sarcopenia Muscle 10(3), 485-500 (2019). 10.1002/jcsm.12411, Pubmed: $\underline{30993881}$ 
289 13. Liu, P. et al. Sarcopenia as a predictor of all-cause mortality among community-

dwelling older people: A systematic review and meta-analysis. Maturitas 103, 16-22

14. De Lorenzo, A., Andreoli, A., Matthie, J. \& Withers, P. Predicting body cell mass with bioimpedance by using theoretical methods: a technological review. J. Appl. Physiol. Bethesda MD 1985 82(5), 1542-1558 (1997). 10.1152/jappl.1997.82.5.1542,

Pubmed:9134904

15. Lukaski, H. C. Evolution of bioimpedance: a circuitous journey from estimation of Pubmed:23299867

16. Norman, K., Stobäus, N., Pirlich, M. \& Bosy-Westphal, A. Bioelectrical phase angle physiological function to assessment of body composition and a return to clinical research. Eur. J. Clin. Nutr. 67 Suppl 1, S2-S9 (2013). 10.1038/ejcn.2012.149,

304 17. Lukaski, H. C., Kyle, U. G. \& Kondrup, J. Assessment of adult malnutrition and prognosis with bioelectrical impedance analysis: phase angle and impedance ratio. Curr. Opin. Clin. Nutr. Metab. Care 20(5), 330-339 (2017). 
308 18. de Blasio, F. et al. Raw BIA variables are predictors of muscle strength in patients with chronic obstructive pulmonary disease. Eur. J. Clin. Nutr. 71(11), 1336-1340 (2017). 10.1038/ejen.2017.147, Pubmed:28901331

311 19. Mundstock, E. et al. Association between phase angle from bioelectrical impedance analysis and level of physical activity: systematic review and meta-analysis. Clin. Nutr. (Edinburgh, Scotland) 38(4), 1504-1510 (2019). 10.1016/j.clnu.2018.08.031, Pubmed: $\underline{30224304}$

20. Di Vincenzo, O., Marra, M., Di Gregorio, A., Pasanisi, F. \& Scalfi, L. Bioelectrical impedance analysis (BIA) -derived phase angle in sarcopenia: A systematic review. Clin. Nutr. (Edinburgh, Scotland) (2020). 10.1016/j.clnu.2020.10.048, Pubmed:33183880

21. Yamada, M. et al. Phase Angle Is a Useful indicator for Muscle Function in Older Pubmed: $\underline{30820513}$ Angle and Sarcopenia in older adults. Nutr. Clin. Pract. Off. Publ. Am. Soc. Parenter. Enter. Nutr. 32(1), 103-109 (2017). 10.1177/0884533616664503, Pubmed:27590205 
23. Espirito Santo Silva, D. D. et al. Phase angle as a marker for sarcopenia in cirrhosis. Clin. Nutr. ESPEN 32, 56-60 (2019). 10.1016/j.clnesp.2019.05.003,

Pubmed: $\underline{31221291}$

24. Kosoku, A. et al. Association of sarcopenia with phase angle and body mass index in kidney transplant recipients. Sci. Rep. 10(1), 266 (2020). 10.1038/s41598-019-57195-

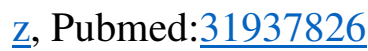

25. Vlaeyen, J. W. S. \& Linton, S. J. Fear-avoidance and its consequences in chronic musculoskeletal pain: a state of the art. Pain 85(3), 317-332 (2000). 10.1016/S03043959(99)00242-0, Pubmed:10781906

26. Asmundson, G. J., Norton, P. J. \& Norton, G. R. Beyond pain: the role of fear and avoidance in chronicity. Clin. Psychol. Rev. 19(1), 97-119 (1999). 10.1016/s02727358(98)00034-8, Pubmed: $\underline{9987586}$

27. Leeuw, M. et al. The fear-avoidance model of musculoskeletal pain: current state of scientific evidence. J. Behav. Med. 30(1), 77-94 (2007). 10.1007/s10865-006-9085-0, Pubmed: $\underline{17180640}$

28. Booth, J. et al. Exercise for chronic musculoskeletal pain: A biopsychosocial approach. Musculoskelet. Care 15(4), 413-421 (2017). 10.1002/msc.1191, Pubmed:28371175 
343 29. O'Connor, S. R. et al. Walking exercise for chronic musculoskeletal pain: systematic review and meta-analysis. Arch. Phys. Med. Rehabil. 96(4), 724-734.e3 (2015).

30. Cruz-Jentoft, A. J. et al. Sarcopenia: revised European consensus on definition and diagnosis. Age Ageing 48(1), 16-31 (2019). 10.1093/ageing/afy169

31. Fielding, R. A. et al. Sarcopenia: an undiagnosed condition in older adults. Current

32. Chen, L. K. et al. Asian Working Group for Sarcopenia: 2019 Consensus Update on Sarcopenia Diagnosis and Treatment. J. Am. Med. Dir. Assoc. 21(3), 300-307.e2 comparison of six methods. Pain 27(1), 117-126 (1986). 10.1016/0304- 
Figures

A

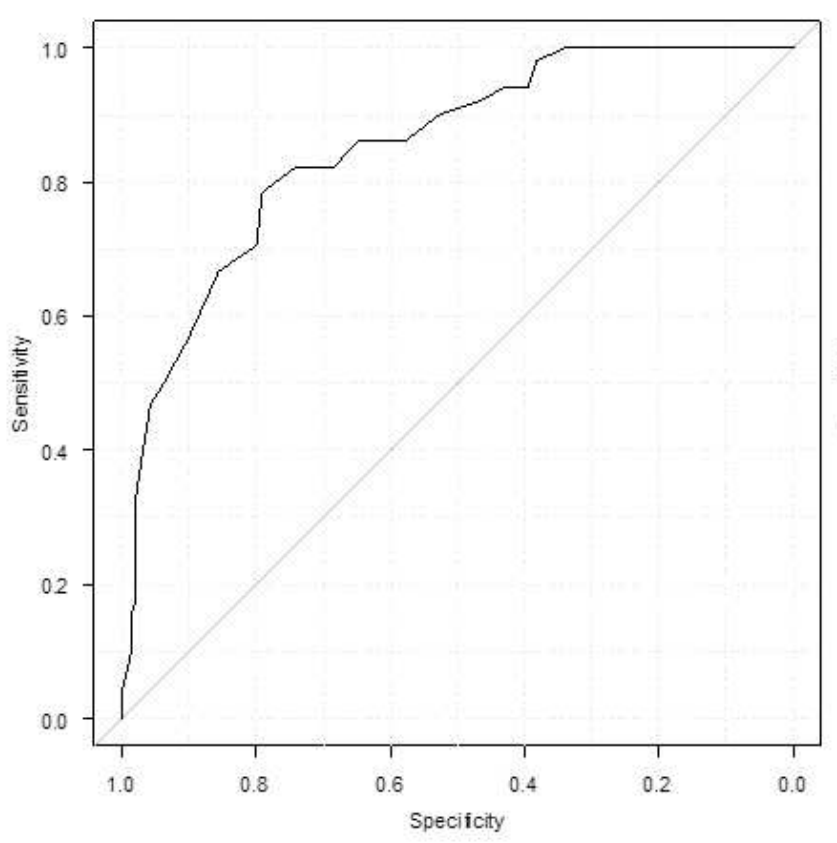

C

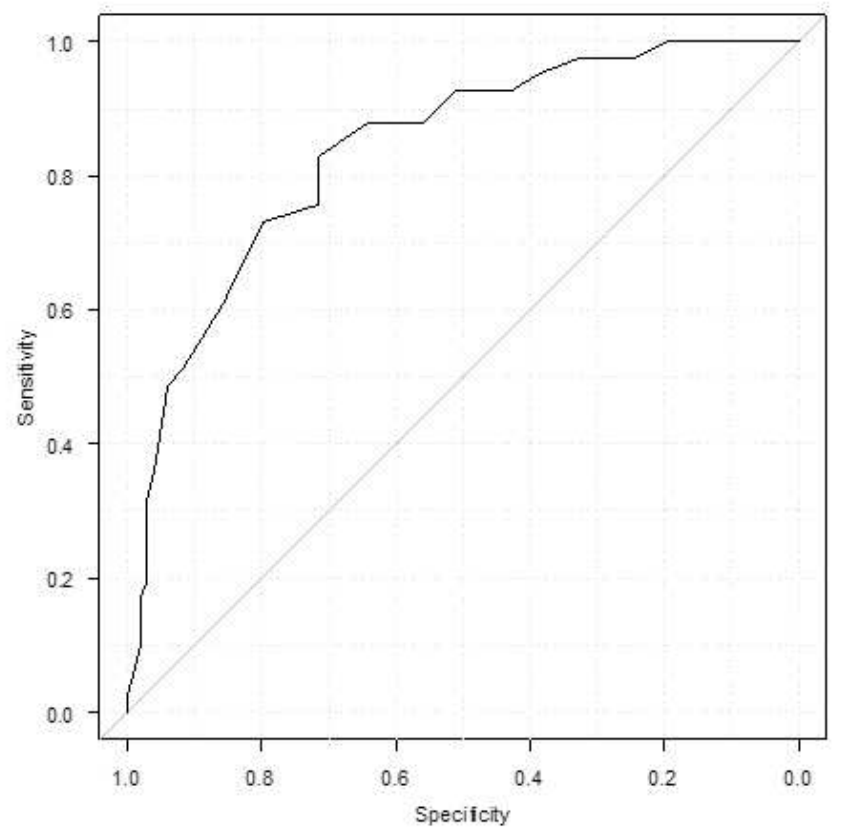

B

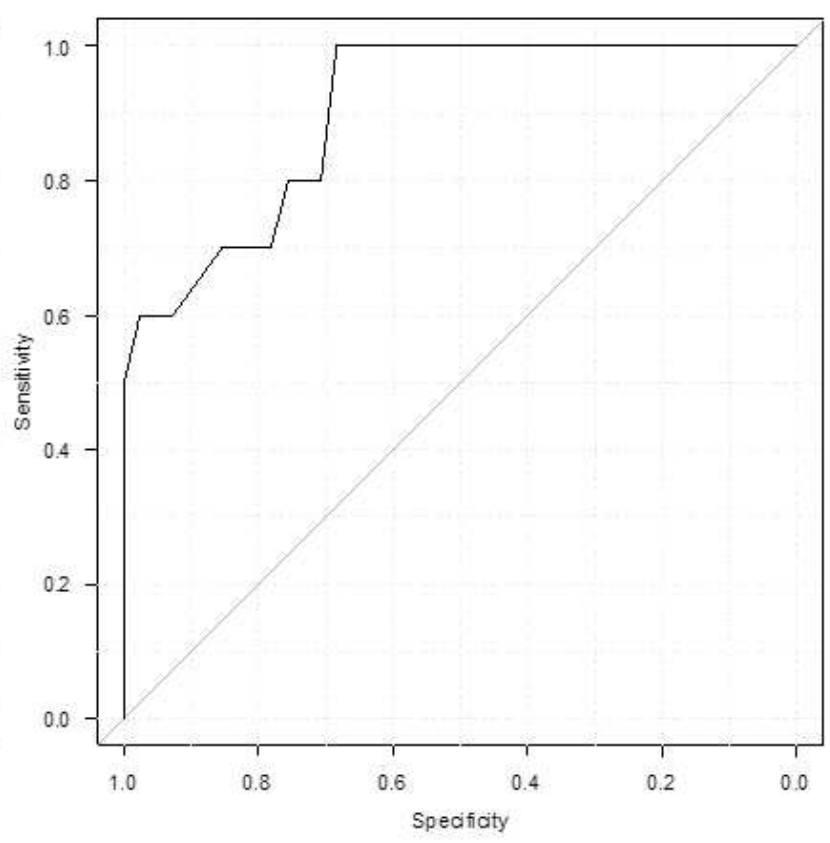

